\title{
Introduction to the special issue on belief revision, argumentation, ontologies, and norms
}

\author{
Eduardo Fermé ${ }^{1}$. Guillermo R. Simari²
}

Published online: 16 November 2019

(C) Springer Nature Switzerland AG 2019

Belief revision is the process of changing beliefs to take into account new information. The last four decades of exploratory inquiry on the Dynamics of Belief has been to logically recover from circumstantial inconsistency, and had produced advances in the understanding of this multifaceted area. Contributing to the study of belief revision are philosophy, formal logic, economics, computer science and artificial intelligence, each with its own different perspectives. Many significant contributions have led to the consolidation of the field in a solid formal structure that supports many exciting proposals of future research lines that stem from that foundation.

Computational Argumentation, on the other hand, represents another area engaged in the study of ascertaining what a reasoning agent could safely assume in the context of conflicting belief. The problems studied in this domain of research have been the concern of philosophers for millennia, and after the formalization of Logic as a distinct domain of inquiry, many matters became defined well enough as to be studied computationally.

This special issue, combines these areas of research intending to achieve a fruitful crossfertilization that will be helpful for both.

Until recently, these two areas of Knowledge Representation have been pursuing their goals along essentially independent research programs. Nevertheless, the task of modeling reasoning processes is an exceedingly challenging, very captivating, and a quite fruitful endeavor for these communities. Belief Dynamics and Argumentation partially capture distinct but surely non-disjoint aspects of the knowledge representation and reasoning challenges, with many opportunities of interchange to address open questions. There exist many places in a possible complex architecture of a reasoning agent where these two areas can work together to obtain substantial results.

Eduardo Fermé

ferme@uma.pt

Guillermo R. Simari

grs@cs.uns.edu.ar

1 Faculty of Exact Sciences and Engineering, University of Madeira, Campus Universitário da Penteada, 9000-390 Funchal, Madeira, Portugal

2 Department of Computer Science and Engineering (DCIC), Institute for Computer Science and Engineering (ICIC), San Andrés 800, Campus Palihue, Bahía Blanca, Argentina 
The five papers contained in this special issue were inspired by discussions during the $3 \mathrm{rd}$ Madeira Workshop on Belief Revision, Argumentation, Ontologies, and Norms (BRAON2017). This collection includes research advanced from the interaction between these areas of research concerned with the epistemic state of an agent. As such, these papers reflect dissimilar perspectives that have contributed to advance the understanding of such a hard and stimulating problem. Our purpose in offering this collection is to reach a wider audience and stimulate research on this compelling topic. We believe that the interaction between Belief Revision and Argumentation will help advance the goals of understanding them separately, and more broadly, understanding the cognitive architecture of a reasoning agent.

In Impossibility Results for Belief Contraction, by Sven Ove Hansson, the author show how three apparently weak and plausible conditions cannot be satisfied by a contraction operator acting on belief sets since they are logically incompatible.

In the survey A Review of the Relations Between Logical Argumentation and Reasoning with Maximal Consistency by Ofer Arieli, AnneMarie Borg and Jesse Heyninck, a selection of results associating Dung-style semantics for different logical argumentation frameworks and particular forms of reasoning with maximally consistent sets of premises. The author also explores the formalisms considering some rationality postulates, carrying the analysis to the corresponding proof systems in nonmonotonic reasoning.

The work presented in Two AGM-Style Characterizations of Model Repair by Paulo T. Guerra and Renata Wassermann, formally examines model repair under the perspective of the AGM theory. Two forms of obtaining model repair are described-one through belief sets an another by means of structural changes. Different sets of postulates are advanced and examined in relation to the intuitive rationality of the problem of model repair and presenting two representation results formulating the connection between them.

In Probability, Coherent Belief and Coherent Belief Change, by John Cantwell and Hans Rott, the authors analyze the statics and dynamics of belief states where these states are formalized as pairs that include the agent's credences and their categorical beliefs; the first is described as a subjective probability measure and the second expressed as a set of possible worlds. This framework is properly examined considering the principles of Inclusion and Preservation for belief revision and the principle of Recovery for belief withdrawals, and the Levi and Harper identities.

Finally, the paper entitled Practical Reasoning Using Values: An Argumentative Approach Based on a Hierarchy of Values by Juan C. L. Teze, Antoni Perelló-Moragues, Lluis Godo and Pablo Noriega, introduces a formal framework based on defeasible argumentation to support the choice of actions of a value-driven agent and arrange these actions into plans matching the agent's preferences. The values the agent considers are assumed as satisfying a partial order in a hierarchy that which is employed in the resolution of conflicts between incommensurable values.

We want to acknowledge and extend our deeply felt appreciation to everyone who has contributed to this issue and to the Madeira Workshop series, particularly the participants, authors, and reviewers. It has become a source of inspiration for the work on the common area that combines Belief Revision and Argumentation. Finally, and distinctly, we also thank the editorial and publishing staff at Springer for their support, especially Martin Golumbic, editor-in-chief of the Annals of Mathematics and Artificial Intelligence.

Publisher's note Springer Nature remains neutral with regard to jurisdictional claims in published maps and institutional affiliations. 